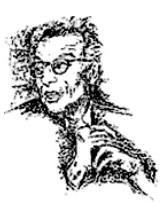

\title{
A FORMULA FOR THE NUMBER OF OVERPARTITIONS OF $n$ IN TERMS OF THE NUMBER OF REPRESENTATIONS OF $n$ AS A SUM OF $r$ SQUARES
}

\author{
Sumit Kumar Jha \\ International Institute of Information Technology, Hyderabad, India \\ kumarjha.sumit@research.iiit.ac.in
}

Received: 7/19/20, Revised: 7/6/21, Accepted: 8/2/21, Published: 8/16/21

\begin{abstract}
Let $\bar{p}(n)$ denote the number of overpartitions of $n$, and $c_{r}(n)$ be the number of representations of $n$ as a sum of $r$ squares where representations with different orders and different signs are counted as distinct. Then we prove that

$$
\bar{p}(n)=\sum_{r=0}^{n}(-1)^{n+r}\left(\begin{array}{l}
n+1 \\
r+1
\end{array}\right) c_{r}(n) .
$$

\section{Main Result}

An overpartition of $n$ is a non-increasing sequence of natural numbers whose sum is $n$ in which the first occurrence of a number may be overlined. Let $\bar{p}(n)$ denote the number of overpartitions of an integer $n$. For convenience, we let $\bar{p}(0)=1$. For example, $\bar{p}(3)=8$ because there are 8 possible overpartitions of 3 :
\end{abstract}

$$
3, \overline{3}, 2+1, \overline{2}+1,2+\overline{1}, \overline{2}+\overline{1}, 1+1+1, \overline{1}+1+1 .
$$

Definition 1. [3, formula 7.324] Let $\theta(q)$ be the following infinite product:

$$
\theta(q):=\prod_{j=1}^{\infty} \frac{1-q^{j}}{1+q^{j}}=\sum_{n=-\infty}^{\infty}(-1)^{n} q^{n^{2}},
$$

where $|q|<1$.

From [1], we have that the generating function of $\bar{p}(n)$ is

$$
\frac{1}{\theta(q)}=\sum_{n=0}^{\infty} \bar{p}(n) q^{n}
$$


Definition 2. For any positive integer $r$ define $c_{r}(n)$ by $\theta(q)^{r}=\sum_{n=0}^{\infty} c_{r}(n)(-1)^{n} q^{n}$ where $c_{r}(n)$ is the number of representations of $n$ as a sum of $r$ squares where representations with different orders and different signs are counted as distinct. For convenience, we let $c_{r}(0)=1$.

Our aim is to derive the following identity.

Theorem 1. For all non-negative integers $n$ we have

$$
\bar{p}(n)=\sum_{r=0}^{n}(-1)^{n+r}\left(\begin{array}{l}
n+1 \\
r+1
\end{array}\right) c_{r}(n) .
$$

We require the following two lemmas for our proof.

Lemma 1. For all positive integers $n$ we have

$$
\bar{p}(n)=\frac{1}{n !} \sum_{k=0}^{n}(-1)^{k} k ! B_{n, k}\left(\tilde{\theta}^{\prime}(0), \tilde{\theta}^{\prime \prime}(0), \ldots, \tilde{\theta}^{(n-k+1)}(0)\right)
$$

where $\tilde{\theta}(q)=\theta(q)-1$ and $B_{n, k} \equiv \mathrm{B}_{n, k}\left(x_{1}, x_{2}, \ldots, x_{n-k+1}\right)$ are the partial Bell polynomials defined by [2, p. 134]

$$
\mathrm{B}_{n, k}\left(x_{1}, x_{2}, \ldots, x_{n-k+1}\right)=\sum_{\substack{1 \leq i \leq n, \ell_{i} \in \mathbb{N} \\ \sum_{i=1}^{n} i \ell_{i}=n \\ \sum_{i=1}^{n} \ell_{i}=k}} \frac{n !}{\prod_{i=1}^{n-k+1} \ell_{i} !} \prod_{i=1}^{n-k+1}\left(\frac{x_{i}}{i !}\right)^{\ell_{i}} .
$$

Proof. Let $f(q)=1 /(1+q)$. Using Faà di Bruno's formula [2, p. 137] we have

$$
f(\tilde{\theta}(q))=\sum_{n=0}^{\infty} h_{n} \frac{q^{n}}{n !}
$$

where $h_{0}=1$ and

$$
h_{n}=\sum_{k=1}^{n} f^{k}(0) B_{n, k}\left(\tilde{\theta}^{\prime}(0), \tilde{\theta}^{\prime \prime}(0), \ldots, \tilde{\theta}^{(n-k+1)}(0)\right) \quad(n \geq 1) .
$$

Since

$$
\frac{d^{n}}{d q^{n}} f(\tilde{\theta}(q))=\frac{d^{n}}{d q^{n}} \frac{1}{\theta(q)}=\frac{d^{n}}{d q^{n}} \sum_{n=0}^{\infty} \bar{p}(n) q^{n}
$$

we have

$$
\begin{aligned}
n ! \bar{p}(n) & =\sum_{k=1}^{n} f^{k}(0) B_{n, k}\left(\tilde{\theta}^{\prime}(0), \tilde{\theta}^{\prime \prime}(0), \ldots, \tilde{\theta}^{(n-k+1)}(0)\right) \\
& =\sum_{k=1}^{n}(-1)^{k} k ! B_{n, k}\left(\tilde{\theta}^{\prime}(0), \tilde{\theta}^{\prime \prime}(0), \ldots, \tilde{\theta}^{(n-k+1)}(0)\right) .
\end{aligned}
$$


Lemma 2. We have, for positive integers $n, k$,

$$
B_{n, k}\left(\tilde{\theta}^{\prime}(0), \tilde{\theta}^{\prime \prime}(0), \ldots, \tilde{\theta}^{(n-k+1)}(0)\right)=(-1)^{n} \frac{n !}{k !} \sum_{r=1}^{k}(-1)^{k-r}\left(\begin{array}{l}
k \\
r
\end{array}\right) c_{r}(n) .
$$

Proof. We start with the generating function for the partial Bell polynomials $[2$, Equation (3a') on p. 133] as follows to conclude (4):

$$
\begin{aligned}
\sum_{n=k}^{\infty} B_{n, k}\left(\tilde{\theta}^{\prime}(0), \tilde{\theta}^{\prime \prime}(0), \ldots, \tilde{\theta}^{(n-k+1)}(0)\right) \frac{q^{n}}{n !} & =\frac{1}{k !}\left(\sum_{j=1}^{\infty} \tilde{\theta}^{(j)}(0) \frac{q^{j}}{j !}\right)^{k} \\
& =\frac{1}{k !}(\theta(q)-1)^{k} \\
& =\frac{1}{k !} \sum_{r=0}^{k}(-1)^{k-r}\left(\begin{array}{c}
k \\
r
\end{array}\right) \theta(q)^{r} \\
& =\frac{1}{k !} \sum_{r=0}^{k}(-1)^{k-r}\left(\begin{array}{c}
k \\
r
\end{array}\right) \sum_{n=0}^{\infty}(-1)^{n} c_{r}(n) q^{n} .
\end{aligned}
$$

Proof of Theorem 1. Combining (2) and (4) we have

$$
\begin{aligned}
\bar{p}(n)=(-1)^{n} \sum_{k=0}^{n} \sum_{r=0}^{k}(-1)^{r}\left(\begin{array}{l}
k \\
r
\end{array}\right) c_{r}(n) & =(-1)^{n} \sum_{r=0}^{n}(-1)^{r} c_{r}(n) \sum_{k=r}^{n}\left(\begin{array}{l}
k \\
r
\end{array}\right) \\
& =(-1)^{n} \sum_{r=0}^{n}(-1)^{r}\left(\begin{array}{l}
n+1 \\
r+1
\end{array}\right) c_{r}(n) .
\end{aligned}
$$

Now we can conclude our main result.

Remark 1. Similar methods have been employed by the author in [4] and [5].

\section{References}

[1] S. Corteel and J. Lovejoy, Overpartitions, Trans. Amer. Math. Soc. 356 (2004), 1623-1635.

[2] L. Comtet, Advanced Combinatorics: The Art of Finite and Infinite Expansions, D. Reidel Publishing Co., Dordrecht, 1974.

[3] N. J. Fine, Basic Hypergeometric Series and Applications, American Mathematical Soc., 1988.

[4] S. K. Jha, A formula for the number of partitions of $\mathrm{n}$ in terms of the partial Bell polynomials, Ramanujan J., 2021, to appear; published online at https://doi.org/10.1007/ s11139-020-00380-w.

[5] S. K. Jha, An identity for the sum of inverses of odd divisors of $n$ in terms of the number of representations of $n$ as a sum of squares, Rocky Mountain J. Math. 51 (2), 581-583. 